\title{
Solving the Kuramoto-Sivashinsky equation via Variational Iteration Method
}

\author{
Majeed A. Yousif *, Saad A. Manaa, Fadhil H. Easif \\ Department of Mathematics, Faculty of Science, University of Zakho,Duhok, Kurdistan Region, Iraq \\ *Corresponding author E-mail: majeed.ahmed@uod.ac
}

Copyright $\odot 2014$ Yousif et al. This is an open access article distributed under the Creative Commons Attribution License, which permits unrestricted use, distribution, and reproduction in any medium, provided the original work is properly cited.

\begin{abstract}
In this study, the approximate solutions for the Kuramoto-Sivashinsky equation by using the Variational Iteration Method (VIM) are obtained. Comparisons with the exact solutions and the solutions obtained by the Homotopy Perturbation Method (HPM), the numerical example show that the Variational Iteration Method (VIM) is accurate and effective and suitable for this kind of problem.
\end{abstract}

Keywords: Kuramoto-Sivashinsky equation, Variational Iteration Method.

\section{Introduction}

It is well known that most of the phenomena that arise in mathematical physics and engineering fields can be described by partial differential equations (PDEs) [1], one of the recently method for solving (PDEs) is Variational Iteration Method, VIM was introduced by Ji-Huan He in 1997.[2] The method has been favorably applied to various kinds of problems; for example, this scheme is used for solving the fractional KdV-Burgers-Kuramoto equation.[3] This technique computes the exact solution of equations using the initial condition only. It is also important to note that the present method does not require discretization of the equation. Therefore, it is not affected by computation round-off errors and one is not faced with the necessity of large computer memory and time. Furthermore, using this idea we do not need to solve any linear or nonlinear system of equations. (VIM) is employed to solve fourth-order parabolic equations. [4] Also, this method is employed to solve delay differential equations. [5]

\section{Mathematical model}

The Kuramoto-Sivashinsky equation was derived by Kuramoto [6] as a model for phase turbulence in reaction diffusion systems and by Sivashinsky [7] as a model for plane flame propagation. This equation describes many kinds of physical phenomenons such as the flow of thin liquid films on inclined planes and dendritic fronts in dilute binary alloys. Boundary control of Kuramoto-Sivashinsky equation has practical applications in engineering. However, even after we linearize the Kuramoto-Sivashinsky equation by dropping the quadratic convective term, the problems of the boundary control of the linearized Kuramoto-Sivashinsky equation are still largely unexplored (see [8]), and there are few results obtained.[9]

Consider the Kuramoto-Sivashinsky equation [10]

$\frac{\partial u}{\partial t}+u \frac{\partial u}{\partial x}+\alpha \frac{\partial^{2} u}{\partial x^{2}}+\gamma \frac{\partial^{3} u}{\partial x^{3}}+\beta \frac{\partial^{4} u}{\partial x^{4}}=0$

Where $\alpha, \gamma$ and $\beta$ are arbitrary constants,

Subject to the initial condition

$u(x, 0)=f(x) \quad a \leq x \leq b$

And boundary conditions

$u(a, t)=g_{1}(t), \quad u(b, t)=g_{2}(t), \quad t \geq 0$. 
And Neumann boundary condition

$\frac{\partial^{2} u}{\partial x^{2}}=h_{1}, \quad$ at $x=a$ and $x=b \quad$ where $h_{1} \geq 0$.

\section{Basic idea of Variational Iteration Method}

To clarify the basic ideas of VIM, we consider the following differential equation

$L u+N u=g(x, t)$

Where $L$ is a linear operator defined by $L=\frac{\partial^{m}}{\partial t^{m}}, m \in \mathbb{N}, N$ is a nonlinear operator and $g(x, t)$ is a known analytic function. According to VIM, we can write down a correction functional as follows:

$u_{n+1}(x, t)=u_{n}(x, t)+\int_{0}^{t} \lambda\left[L u_{n}(x, \tau)+N \tilde{u}_{n}(x, \tau)-g(x, \tau)\right] d \tau$

Where $\lambda$ is a general lagrangian multiplier defined as: [11]

$\lambda(t, \tau)=\frac{(-1)^{m}}{(m-1) !}(\tau-t)^{m-1} \quad, m \geq 1$.

The subscript $n$ indicates the $n$th approximation and $\tilde{u}_{n}$ is considered as a restricted variation. [2]

\section{Derivative of (VIM) for Kuramoto-Sivashinsky equation}

To solve Kuramoto-Sivashinsky equation (1), with initial condition (2) by means of VIM, we construct a correction functional:

$u_{n+1}(x, t)=u_{n}(x, t)+\int_{0}^{t} \lambda\left(\frac{\partial u_{n}(x, \tau)}{\partial \tau}+u_{n}(x, \tau) \frac{\partial \widetilde{u}_{n}(x, \tau)}{\partial x}+\alpha \frac{\partial^{2} \widetilde{u}_{n}(x, \tau)}{\partial x^{2}}+\gamma \frac{\partial^{3} \widetilde{u}_{n}(x, \tau)}{\partial x^{3}}+\beta \frac{\partial^{4} \widetilde{u}_{n}(x, \tau)}{\partial x^{4}}\right) d \tau$

In our equation, $m=1$, then by formula (7), $\lambda=-1$ substituting in equation (8) we get:

$u_{n+1}(x, t)=u_{n}(x, t)-\int_{0}^{t}\left(\frac{\partial u_{n}(x, \tau)}{\partial \tau}+u_{n}(x, \tau) \frac{\partial u_{n}(x, \tau)}{\partial x}+\alpha \frac{\partial^{2} u_{n}(x, \tau)}{\partial x^{2}}+\gamma \frac{\partial^{3} u_{n}(x, \tau)}{\partial x^{3}}+\beta \frac{\partial^{4} u_{n}(x, \tau)}{\partial x^{4}}\right) d \tau, n=0,1, \ldots$

We start with the initial approximation of $u(x, 0)$ given by equation (2). Using the iteration formula (9), we can obtain the other components as follows:

$u_{0}(x, t)=u(x, 0)=f(x)$.

For $n=0$;

$u_{1}(x, t)=u_{0}(x, t)-\int_{0}^{t}\left(\frac{\partial u_{0}(x, \tau)}{\partial \tau}+u_{0}(x, \tau) \frac{\partial u_{0}(x, \tau)}{\partial x}+\alpha \frac{\partial^{2} u_{0}(x, \tau)}{\partial x^{2}}+\gamma \frac{\partial^{3} u_{0}(x, \tau)}{\partial x^{3}}+\beta \frac{\partial^{4} u_{0}(x, \tau)}{\partial x^{4}}\right) d \tau$

For $n=1$;

$u_{2}(x, t)=u_{1}(x, t)-\int_{0}^{t}\left(\frac{\partial u_{1}(x, \tau)}{\partial \tau}+u_{1}(x, \tau) \frac{\partial u_{1}(x, \tau)}{\partial x}+\alpha \frac{\partial^{2} u_{1}(x, \tau)}{\partial x^{2}}+\gamma \frac{\partial^{3} u_{1}(x, \tau)}{\partial x^{3}}+\beta \frac{\partial^{4} u_{1}(x, \tau)}{\partial x^{4}}\right) d \tau$

And by the same way for $n=2,3, \ldots$

\section{Applications}

In this section, we have solved the Kuramoto-Sivashinsy equation numerically by using Variational Iteration Method (VIM). For clarifying, we take the following example:

\section{Example:}

Consider Kuramoto-Sivashinsky equation by [12]

$u_{t}+u u_{x}+u_{x x}+u_{x x x x}=0, \quad x \in[0,32 \pi], \quad t \in[0,0.001]$;

With the initial condition

$u(x, 0)=\cos \left(\frac{x}{16}\right)\left(1+\sin \left(\frac{x}{16}\right)\right)$,

And the exact solution of the problem is given by

$u(x, t)=\cos \left(\frac{x}{16}-t\right)\left(1+\sin \left(\frac{x}{16}-t\right)\right)$.

For solving by (VIM) we obtain the recurrence relation

$u_{n+1}(x, t)=u_{n}(x, t)-\int_{0}^{t}\left(\frac{\partial u_{n}(x, \tau)}{\partial \tau}+u_{n}(x, \tau) \frac{\partial u_{n}(x, \tau)}{\partial x}+\frac{\partial^{2} u_{n}(x, \tau)}{\partial x^{2}}+\frac{\partial^{4} u_{n}(x, \tau)}{\partial x^{4}}\right) d \tau, \quad n=0,1, \ldots$

Starting with the initial approximation

$u_{0}(x, t)=\cos \left(\frac{x}{16}\right)\left(1+\sin \left(\frac{x}{16}\right)\right)$ 


$$
\begin{aligned}
& u_{1}(x, t)=\cos \left(\frac{x}{16}\right)\left(1+\sin \left(\frac{x}{16}\right)\right)+t\left(\frac{8447 \cos \left(\frac{x}{16}\right)}{65536}-\frac{\cos ^{3}\left(\frac{x}{16}\right) \sin \left(\frac{x}{16}\right)}{8}-\frac{3 \cos ^{3}\left(\frac{x}{16}\right)}{16}+\frac{575 \sin \left(\frac{x}{16}\right) \cos \left(\frac{x}{16}\right)}{4096}\right) \\
& u_{2}(x, t)=\cos \left(\frac{x}{16}\right)\left(1+\sin \left(\frac{x}{16}\right)\right)+t\left(\frac{3839 \cos \left(\frac{x}{16}\right)}{32768}-\frac{\cos ^{3}\left(\frac{x}{16}\right) \sin \left(\frac{x}{16}\right)}{8}-\frac{3 \cos ^{3}\left(\frac{x}{16}\right)}{16}+\frac{575 \sin \left(\frac{x}{16}\right) \cos \left(\frac{x}{16}\right)}{4096}-\frac{\sin \left(\frac{x}{4}\right)}{64}-\right. \\
& \left.\frac{3 \cos \left(\frac{3 x}{16}\right)}{64}+\frac{319 \sin \left(\frac{x}{8}\right)}{8192319}\right)+t^{2}\left(\frac{25 \cos \left(\frac{5 x}{16}\right)}{4096}-\frac{7780863 \cos \left(\frac{x}{16}\right)}{4294967296}-\frac{6765 \cos \left(\frac{3 x}{16}\right)}{2097152}-\frac{1207 \sin \left(\frac{x}{4}\right)}{131072}-\frac{119199 \sin \left(\frac{x}{8}\right)}{33554432}+\frac{3 \sin \left(\frac{3 x}{8}\right)}{2048}\right)+ \\
& t^{3}\left(\frac{440637 \cos \left(\frac{3 x}{16}\right)}{17179869184}+\frac{34435 \cos \left(\frac{5 x}{16}\right)}{134217728}-\frac{1127873 \cos \left(\frac{x}{16}\right)}{17179869184}-\frac{21 \cos \left(\frac{7 x}{16}\right)}{131072}-\frac{\sin \left(\frac{x}{2}\right)}{32768}-\frac{27937 \sin \left(\frac{x}{4}\right)}{1073741824}+\frac{89601 \sin \left(\frac{x}{8}\right)}{137438953472}+\frac{2685 \sin \left(\frac{3 x}{8}\right)}{8388608}\right)
\end{aligned}
$$

Then by the same way for $u_{3}(x, t), u_{4}(x, t), \ldots$

Table 1: Absolute Error of (HPM) and (VIM) At $3^{\text {rd }}$ Order, When $t=0.0004$

\begin{tabular}{ccc}
\hline$x \times \pi$ & $\left|u_{\text {exact }}-u_{H P M}\right|$ & $\left|u_{\text {exact }}-u_{V I M}\right|$ \\
\hline 0 & $3.766364391323274 \times 10^{-4}$ & $3.297496586894821 \times 10^{-4}$ \\
6.4 & $6.750910825057410 \times 10^{-4}$ & $6.174481700532697 \times 10^{-4}$ \\
12.8 & $1.248599280267992 \times 10^{-4}$ & $1.511274889944847 \times 10^{-4}$ \\
19.2 & $3.679513787964717 \times 10^{-4}$ & $3.862367434608327 \times 10^{-4}$ \\
25.6 & $5.536563414442614 \times 10^{-5}$ & $5.260390638544416 \times 10^{-5}$ \\
32 & $3.766364391323274 \times 10^{-4}$ & $3.297496586894821 \times 10^{-4}$ \\
\hline
\end{tabular}

Table 2: Absolute Error of (HPM) and (VIM) At $3^{\text {rd }}$ Order, when $t=0.0008$

\begin{tabular}{ccc}
\hline$x \times \pi$ & $\left|u_{\text {exact }}-u_{H P M}\right|$ & $\left|u_{\text {exact }}-u_{V I M}\right|$ \\
\hline 0 & $7.534327929941131 \times 10^{-4}$ & $6.596600858967960 \times 10^{-4}$ \\
6.4 & $1.349941768170049 \times 10^{-3}$ & $1.234642967767430 \times 10^{-3}$ \\
12.8 & $2.501510819914454 \times 10^{-4}$ & $3.026732222584094 \times 10^{-4}$ \\
19.2 & $7.360770433769148 \times 10^{-4}$ & $7.726453090434737 \times 10^{-4}$ \\
25.6 & $1.105927804877852 \times 10^{-4}$ & $1.050693972385001 \times 10^{-4}$ \\
32 & $7.534327929941131 \times 10^{-4}$ & $6.596600858967960 \times 10^{-4}$ \\
\hline
\end{tabular}
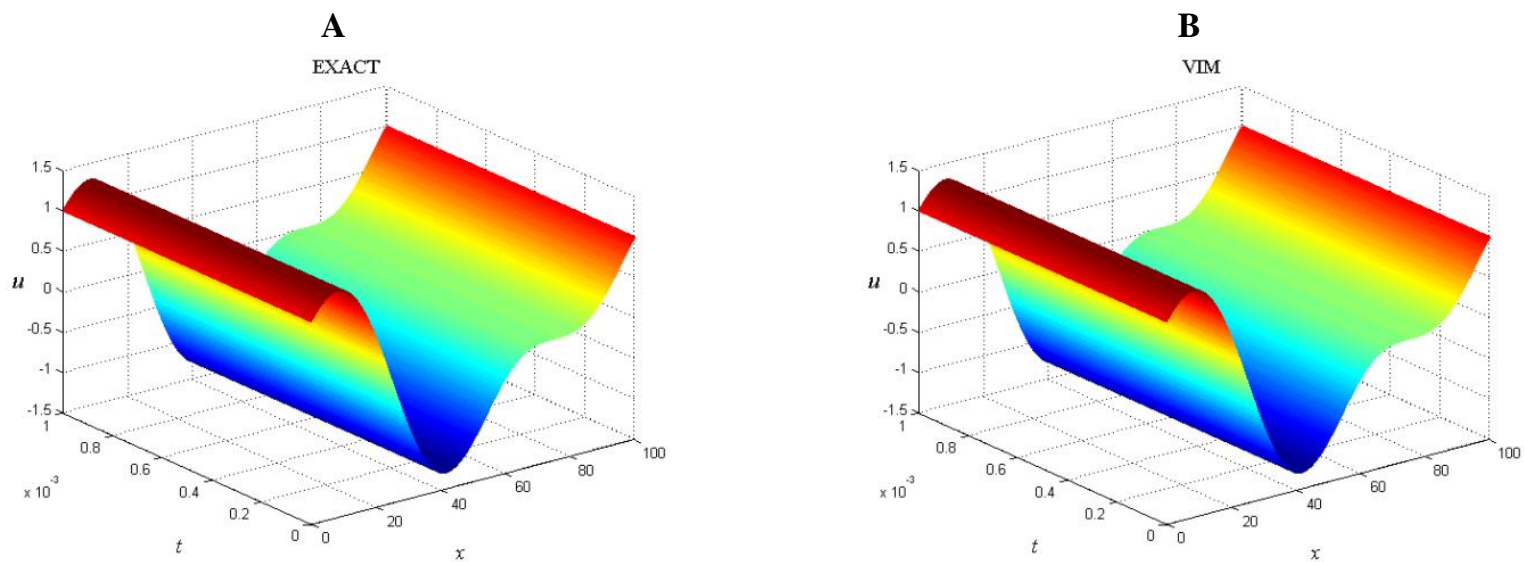

Fig. 1: The Surface shows the solution $u(x, t)$, when $x \in[0,32 \pi], t \in[0,0.001]$ : (A) Exact solution, (B) 3rd Order of approximate solution (VIM)
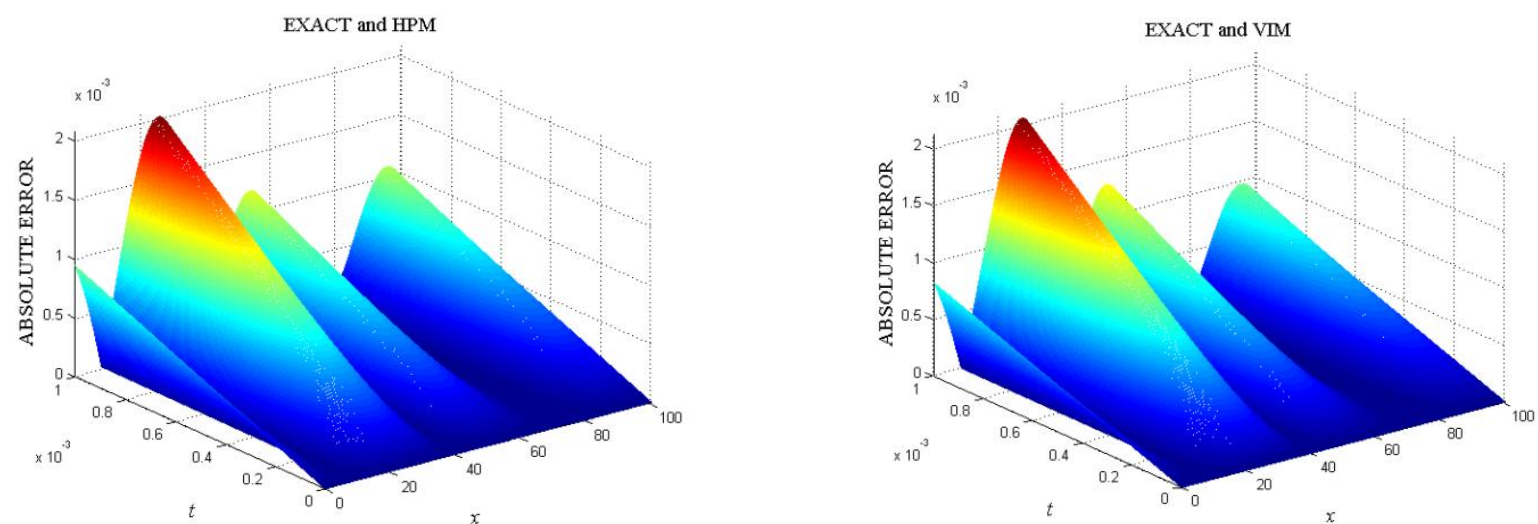

Fig. 2: Absolute error of exact solution, when $x \in[0,32 \pi], t \in[0,0.001]$ and: (A) $3^{\text {rd }}$ Order of (HPM), (B) $3^{\text {rd }}$ Order of (VIM) approximate solution 
A

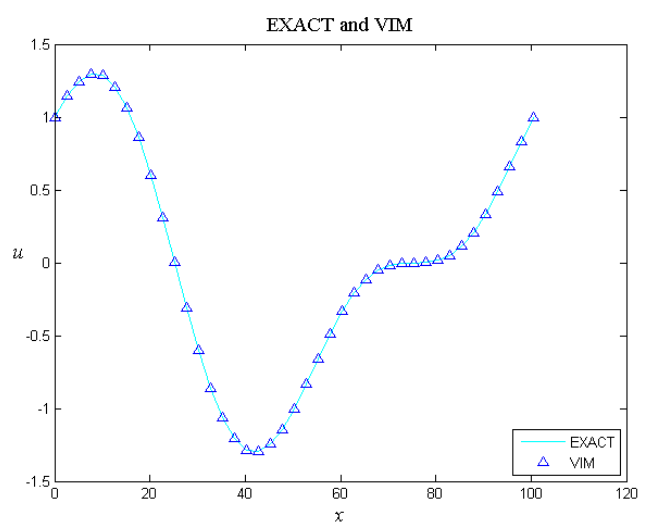

B

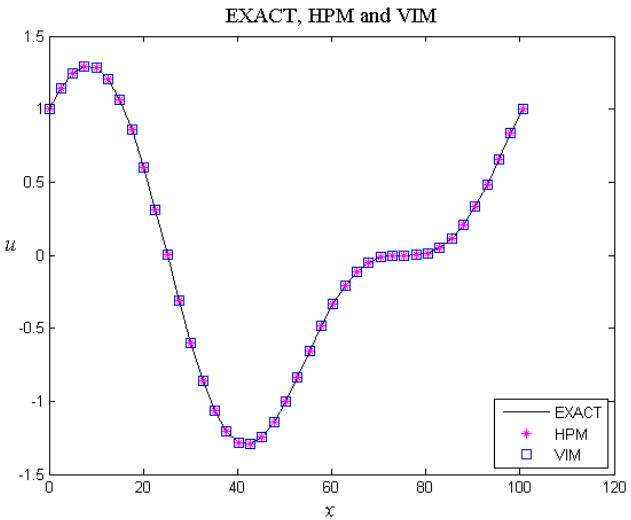

Fig. 3: The curve shows the solution $u(x, t)$, when $x \in[0,32 \pi], t=0.0004$ : (A) Exact solution and $3^{\text {rd }}$ Order of (VIM), (B) Exact Solution, and $3^{\text {rd }}$ Order of (HPM) and $3^{\text {rd }}$ Order of (VIM).
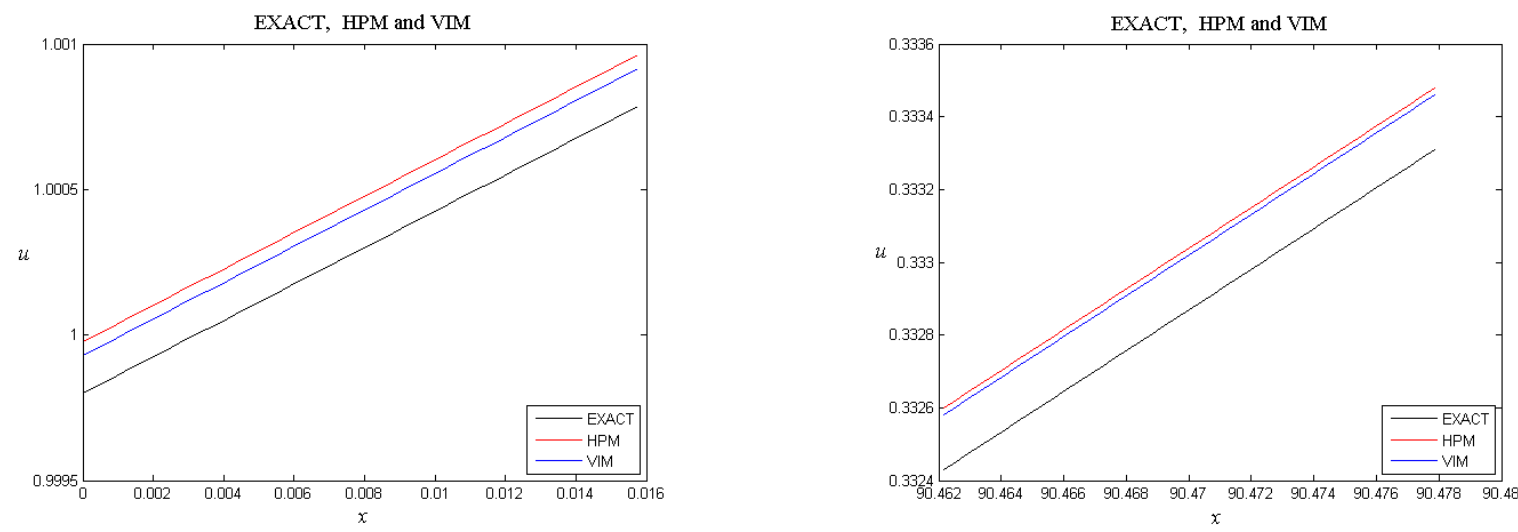

Fig. 4: The zoom curve shows the solution $u(x, t)$ of exact solution, $3^{\text {rd }}$ Order of (HPM) and $3^{\text {rd }}$ Order of (VIM): (A) when $x \in[0,0.005 \pi]$, $t=0.0004,(\mathbf{B})$ when $x \in[28.795 \pi, 28.8 \pi], t=0.0004:$ :
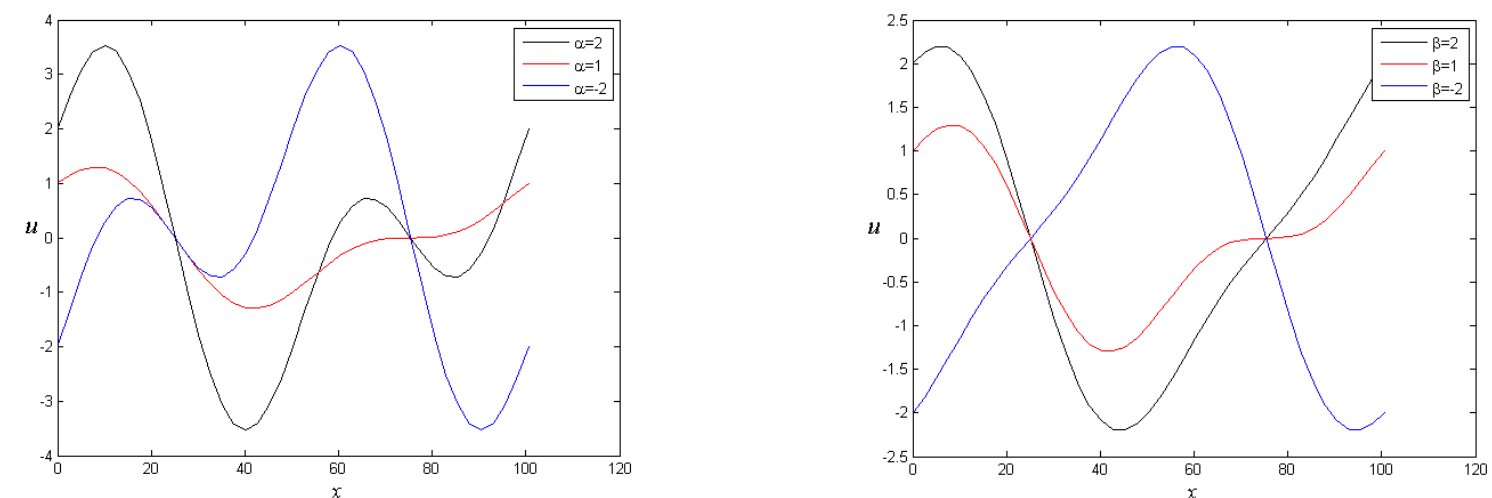

Fig. 5: The curve shows the solution $u(x, t)$ By $3^{\text {rd }}$ Order of (VIM), when $\in[0,32 \pi], t=0.0004:(\mathbf{A})$ when $\alpha=-2, \alpha=1, \alpha=2$, and $\beta=1$ (B) when $\beta=-2, \beta=1, \beta=2$, and $\alpha=1$.

\section{Conclusion}

The Variational Iteration Method (VIM) applied to Kuramoto-Sivashinsky equation and comparing $3^{\text {rd }}$ order of VIM with the exact solution and $3^{\text {rd }}$ order of Homotopy Perturbation Method (HPM) those obtained by Fadhil [13], by Fig.1, Fig.2, Fig.3 and Fig.4, and the absolute error between them in Table1 and Table2. Show that the Variational Iteration Method (VIM) is more accurate and the absolute error is so small and the approximate solution is so closed to the exact solution, Fig.5 show that $\alpha, \beta$ are so effective in this model. 


\section{Acknowledgements}

Many thanks to University of Zakho for supporting this work.

\section{References}

[1] A. M. Wazwaz, Partial Differential Equations and Solitary Waves Theory, Springer, (2009).

[2] J. H. He,"A new approach to nonlinear partial differential equations", Communications in Nonlinear Science and Numerical Simulation, 2, (1997), pp. 230-235.

[3] M. Safari, D. D. Ganji," Application of He's variational iteration method and Adomian's decomposition method to the fractional, KdVBurgers-Kuramoto equation", Computers and Mathematics with Applications, 58, (2009), pp. 2091-2097.

[4] J. Biazar, H. Ghazvini," He's variational iteration method for fourth-order parabolic equations", Computers and Mathematics with Applications, 54, (2007), pp.1047-1054.

[5] J. H. He," Variational iteration method for delay differential equations ", Communications in Nonlinear Science and Numerical Simulation, 2 , (1997), pp. 236-237.

[6] Y. Kuramoto, "Diffusion-induced chaos in reaction systems", Suppl. Prog. Theor. Phys, 64, (1978), pp. 346-367.

[7] G. I. Sivashinsky, "Nonlinear analysis of hydrodynamic instability in laminar flames I: Derivation of basic equations", Acta Astronau, 4, (1977), pp. 1177-1206.

[8] M. Krstic, Boundary control of PDEs: A Course on Backstepping Designs,Philadelphia: Society for Industrial and Applied Mathematics, 2008, chapter 10.

[9] Z. Shengtao, X. Chengkang, L. Guoping," Output-feedback Stabilization of the Kuramoto-Sivashinsky-like Equation”, The 30th Chinese Control Conference, (2011), pp. 1015-1020.

[10] A. M. Wazwaz, "New solitary wave solutions to the Kuramoto-Sivashinsky and the Kawahara equations", Applied Mathematics and Computation, 182, (2006), pp. 1642-1650.

[11] G. CH. Wu, "Challenge in the Variational iteration method -A new approach to identification of the Lagrange multipliers", Journal of King Saud University, 25, (2013), pp. 175-178.

[12] A. Kassam, L. Trefethen, "Fourth-Order Time-Stepping for Stiff PDEs", SIAM J. SCI. COMPUT. , 26, (2005), pp. 1214-1233.

[13] F. H. Easif, S. A. Manaa, Majeed A. Yousif, "The Homotopy Perturbation Method for Solving the Kuramoto-Sivashinsky equation", IOSR Journal of Engineering, 3,(2013), pp. 22-27. 